\title{
Bohumil Cenkl
}

The elliptic differential operators

Commentationes Mathematicae Universitatis Carolinae, Vol. 8 (1967), No. 2, 175--197

Persistent URL: http://dml.cz/dmlcz/105103

\section{Terms of use:}

(C) Charles University in Prague, Faculty of Mathematics and Physics, 1967

Institute of Mathematics of the Academy of Sciences of the Czech Republic provides access to digitized documents strictly for personal use. Each copy of any part of this document must contain these Terms of use.

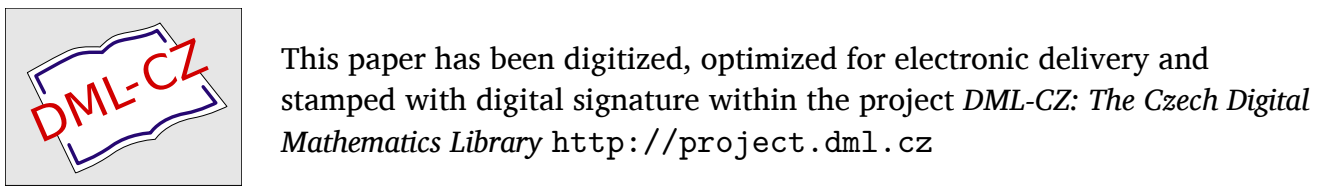




\section{Commentationes Mathematicae Universitatis Carolinae}

$$
8,2 \text { (1967) }
$$

\section{THE EILIPTIC DIFFERENTIAL OPERATORS}

$$
\text { Bohumil CENKL, Praha }
$$

1. Sufficient conditions for vanishing of the cohomology groups, of a complex compact manifold $M$, with values in the sheaf of germs of holomorphic sections of a complex line bundle over $M$ were given by K.Kodaira [4]. The conditions are formulated in terms of the characteristic class of a complex line bundle over M. In this paper a generalization of this problem is solved for a regular elliptic system of linear partial differential equations on a compact differentiable manifold $M$. The condition for vanishing of the cohomology groups $H^{n}(M, \Theta), n>0, \Theta$ being the sheaf of germs of solutions of a homogeneous regular elliptic system $D$ is stated in terms of sufficient positivity of the curvature of the operator $D$.

The Spencer's resolution of $\Theta$ by sheaves of germs of jet forms $C^{r}$, exactness of which is assumed, can be simplified in some sense. We get the so-called " $\beta$-resolution

$$
0 \rightarrow \Theta \rightarrow \underline{B^{0}} \stackrel{B D_{0}}{\longrightarrow} \underline{B}^{1} \stackrel{B D_{0}}{\longrightarrow} \ldots \stackrel{B D_{0}}{\longrightarrow} \underline{B}^{n} \rightarrow 0
$$

where $B$ are differential form and $\beta D_{0}$ is a first order differential operator. This resolution is equivalent to the original Spencer's resolution. Sufficient conditions 
for vaniahing of $H^{n}(M, \Theta), n>0$ are then given in terms of the $\beta$-sequence.

Considering the manifold $M$ and the respective complex analytic vector bundies we get, on the basis of the AtiyahSinger index theorem and the vanishing theorem, relation between the dimension of the space of global solutions of the homogeneous system and the topological index of some elliptic differential operator assiociated to the origiral operator.

The exactress of the resolutions of $\Theta$ is closely related to the existence problem for over-determined systems of elliptic differential equations, $D_{s}=f$ (see [6]). The exactness itself can be studied in connection with a 10cal D-Neumann problem [8],[9]. The fundamental estimate for the Dirichlet integral $Q(\mu, \mu)=\|D u\|^{2}+\left\|D^{*} u\right\|^{2}+\|u\|^{2}$ $\left(u\right.$ is a section of $\left.C^{n}, \mu>0\right)$ is required for the solvability of the D-Neumann problem for a finite submanifold $M$ of a $C^{\infty}$-manifold $M^{\prime}$. The curvature of the operator $D$ allows to give an explicit expression for the Dirichlet integral, and also some sufficient conditions for vanishing of the cohomology groupe $H^{n}=Z\left(\mathbb{C}^{*}\right) / D\left(\mathbb{C}^{n+1}\right)$ in positive degrees. Here $Z\left(\mathbb{C}^{n}\right)$ is the kernel of the map $D$ : $\mathbb{C}^{n} \rightarrow \mathbb{C}^{n+1}$, where $\mathbb{C}^{n}$ denotes the space of sections of $\mathbb{C}^{n}$ over $M$ which are smooth up to the boundary of $M$.

Details and complete proofs will be given in the paper "Vaniahing theorem for an elliptic differential operator" in the Pacific J.Math. - This work was done during the author's stay at Stanford University. -

2. We consider only manifolds, vector bundles and maps of these objects which are "smooth", 1.e. $c^{\infty}$. The sheaf of 
germs of smooth sections of a vector bundle $E$ will be denoted by $E$. Let $M$ be an $n$-dimensional manifold and $E$, $P$ be vector bundles of dimensions $m, l$ respectively, over $M$. We denote by $J_{\mu}(E) \rightarrow M$ the bundle of $\mu-$

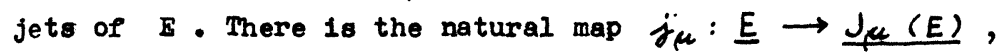
which to section $s$ of $E$ and point $x \in M$ associates the $\mu$-jet of at $x$. Denoting by $S^{\mu}\left(T^{*}\right)$ the $\mu$-th symmetric product we see that $E^{\prime} \otimes S^{\mu+1}\left(T^{*}\right)$ is the kernel of the natural projection $\pi: J_{\mu+1}(E) \rightarrow J_{\mu}(E)$. Let us donote $E^{r}=E \otimes \wedge^{n} T^{*}$ for any vector bundle $E$. There exists a first order dirferential operator $D: J_{\mu+1}(E) \longrightarrow J_{\mu}^{1}(E)$ such that for any smooth function $f$ on $M$ and $\sigma \in J_{\mu+1}(E)$ holds $D f \sigma=d f \otimes \pi \sigma+f D \sigma$.

And $D \sigma=0$ if and only if $\sigma=j_{\mu} s$ for some $s \in \underline{E}$. The operator $D$ extends uniquely to a "derivation" $D: J_{\mu+1}^{\kappa}(E) \rightarrow J_{\mu}^{n+1}(E)$. Then $D^{2}=0$. The operat or $D$ gives rise to the formal differential $\sigma$. The operator $\sigma$ is defined in such a way that the following diagram is commutative. (1)

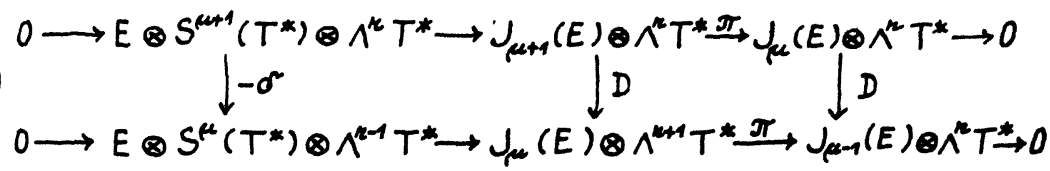

Locally in the coordinate neighborhood $U$ on $M$, with coordinates $x=\left(x^{1}, \ldots, x^{n}\right)$ a local section $\sigma \epsilon$ $\epsilon J_{\mu+1}^{n}(E)$ over $U$ can be expressed as follows: $\sigma=$ $=\left\{\sigma_{q}|| q \mid \leq \mu+1\right\}$, where $q=\left(q_{1}, \ldots, q_{n}, \ldots, q_{n}\right)$ is an ordered n-tuple of non-negative integers $q_{k},|q|=q_{1}+\ldots$ $\ldots+q_{n} ; \sigma_{q}=\left\{\sigma_{q}^{j} \mid 1 \leqslant j \leqslant m\right\}$, where 


$$
\sigma_{2}^{j}=\frac{1}{n !} \sum \sigma_{Q, i_{1}, \ldots i_{n}}^{j}
$$

The formal differential $\sigma$ of $\sigma$ is given by

$$
(\delta \sigma)_{2}=\sum_{j=1}^{n} d x^{j} \wedge \sigma_{q+1_{j}},
$$

and the operator $D$ applied to $\sigma$ has the form $D \sigma=$ $=d \pi \sigma-\delta \sigma$.

A sheaf map $D: E \rightarrow E$, which is in trivializations of the bundles $E$ and $F$ over the coordinate neighborhood $U$ given by the formula

$$
D_{s}=\sum_{|\alpha| \leq \mu_{0}} a_{\alpha} D^{\alpha} s, \quad \alpha=\left(\alpha_{1}, \ldots, \alpha_{m}\right),
$$

$D^{\alpha}=\frac{\partial^{|\alpha|}}{\left(\partial x^{1}\right)^{\alpha_{1}} \ldots\left(\partial x^{m}\right)^{\alpha_{m}}}$, s $\in E \mid U, a_{\alpha}$ are $(l \times m)-$ mat$r i x$-valued functions, is said to be a differential operator of order $\mu_{0}$ from $F$ to $F$. There exists a unique bundle map $\rho \mu_{0}+\nu$, for each $\nu \geqq 0$, which makes the diagram

(2)

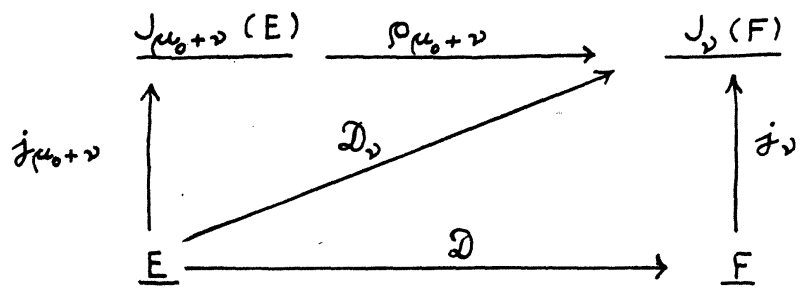

commutative. The operator $D_{2}$, is the so-called $\nu$-th prolongation of $D$.

Definition 1. A regülar system of partial differential equations of order $\mu_{0}$ given by $D$ on $M$ is the kernel $R_{\mu_{0}}$ of the map $\int_{\mu_{0}}$ in the exact sequence 


$$
0 \longrightarrow R_{\mu_{0}} \longrightarrow J_{\mu_{0}}(E) \stackrel{\rho_{\mu_{0}}}{\longrightarrow} F
$$

if

(1) the $R \mu$ are vector bundles over $M$,

(ii) the map $\pi: R_{\mu+1} \longrightarrow R_{\mu}$ is surjective for $\mu \geqq \mu_{0}$.

A smooth section $f \in \underline{E}$ such that $j_{\mu_{0}} f \in \underline{R_{\mu_{e}}}$ is called a solution of the system $R \mu_{0}$.

If the conditions ( $i$ ) and ( $i$ ) are satisfied we speak about the regular operator $D$, and we shall study such operators only.

To the differential operator $D$ and any $\xi \in T_{x}^{*}$, $x \in M, \xi \neq 0$ there is defined the symbol $\sigma(D, \xi)$ : $E_{x} \rightarrow F_{x}$ of $D$ as the composed map $E_{x} \stackrel{x}{\rightarrow} E_{x} \otimes S^{\mu_{0}}\left(T_{x}^{*}\right) \longrightarrow J_{\mu_{0}}(E)_{x} \stackrel{\rho_{\mu_{e}}}{\longrightarrow} F_{x}$. Locally for some $e \in E_{x}$. We have

$$
\sigma(D, \xi) e=\sum_{|\alpha|=\mu_{0}} a_{\alpha}(x) \xi^{\alpha} e .
$$

The exact sequence

$$
0 \rightarrow q_{\mu}^{*} \longrightarrow R_{\mu}^{\kappa} \stackrel{\pi}{\longrightarrow} J_{\mu-1}^{n}(E) \longrightarrow 0
$$

defines for $\mu \geq \mu_{0}$ the vector bundles $g_{\mu}^{n}, \kappa=$ $=0,1, \ldots, n ; \mu \geq \mu_{0}$.

The sequence

(3) $0 \rightarrow g_{\nu+n} \stackrel{\delta^{\sigma}}{\longrightarrow} g_{\nu+n-1}^{1} \stackrel{\delta^{\alpha}}{\longrightarrow} \ldots \stackrel{\delta^{\alpha}}{\longrightarrow} g_{\nu}^{n} \rightarrow 0, \nu \geqq \mu_{0}$

is not exact in general; but we st1ll have $\sigma^{2}=0$.

The corresponding cohomology $H^{*}(g)$ is called $\delta^{r}$-cohomology. The cohomology groups $H^{\mu, \kappa}(g)$ vanish for $\nu \geqq$ $\geqq \mu_{0}+1, \mu=0,1,2, \ldots \quad$ if and only if the sequence 
(3) Is exact.

The system of regular partial differential equations $B_{\mu}$. (or the operator $D_{\mu_{0}}$ ) is said to be involutive if the sequence (3) is exact. The invalutioness defined in this way is that one used by E.Cartan, as was proved by J.P.Serre [7].

3. Assume that $\mu \geqq \mu_{1}$ i.e. $H^{\mu, n}(g)=0$, and define the vector bundles.

$$
A_{\mu}^{n}=\left\{\xi \in g_{\mu}^{n} \mid \delta \xi=0\right\}, \quad C_{\mu}^{n}=R_{\mu+1}^{n} / A_{\mu+1}^{n}
$$

for all non-negative integers $r$. The sections of $C_{\mu}^{n}$ over $M$ are called jet-forms. The commutative diagram

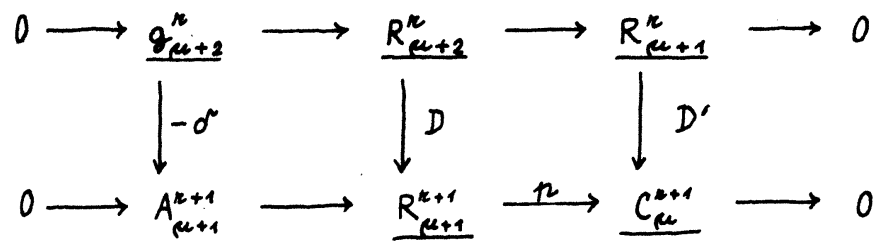

defines the first-order operator $D^{\prime}: R_{\mu+1}^{N} \longrightarrow C_{\mu}^{k+1}$ which factors through $C_{\mu}^{\kappa}$, so that we have the first order differential operator $D: \frac{C_{\mu}^{N}}{\longrightarrow} \frac{C_{\mu}^{N+1}}{\mu}$ such that $D^{\prime}=D$ p. We get then the Spencer's resolution

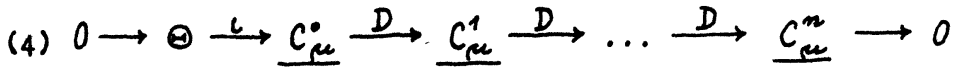

the exactness of which is a non-trivial problem discussed later. Straightforward calculation gives an explicit description of $C_{\mu}^{n}$.

Proposition 1. Each element $\mu \in C_{\mu}^{\mu}, \mu \geq \mu_{1}, \mu \geqq 0$ can be represented as a pair $(\sigma, \xi) \in R_{\mu}^{n} \oplus A_{\mu}^{n+1} \quad$ such that $\sigma=\pi \rho, \xi=\delta \rho$ for some elemènt $\rho \in R_{\mu+1}^{\mu}$, and 
$D_{u}=(d \sigma-\xi,-d \xi) ; D^{2}=0$.

If the adjoint of the operator $D$ (with respect to some motric) is to be considered it is useful to give representation of the elements of $\mathbf{C}_{\mu}^{\kappa}$ by pairs of independent elements. Proposition 2. To a given splitting $\lambda: R_{\mu}^{n} \longrightarrow R_{\mu+1}^{\mu}$ of the exact sequence

$$
0 \longrightarrow g_{\mu+1}^{n} \longrightarrow R_{\mu+1}^{n} \longrightarrow R_{\mu}^{\mu} \longrightarrow 0
$$

there corresponds an isomorphism $C_{\mu}^{k} \cong R_{\mu}^{n} \oplus A_{\mu}^{\mu+1}$, and $D u=\left(D_{0} \sigma-\xi, D_{0}\left(D_{0} \sigma-\xi\right)\right)$, where $\mu=$

$=(\sigma, \xi) \in R_{\mu}^{n} \oplus A_{\mu}^{n+1}, D_{0}=\alpha-\sigma \lambda, D^{2}=0$.

Let us introduce a riemannian metric along the fibres of the vector bundle $\mathbb{B}_{\mu}$ and also some riemannian metric along the fibres of $T(M)$.

We have then on $R_{\mu}^{n}, \quad \mu=0,1, \ldots, n$ the inner product $<,\rangle_{x}, x \in M$ and the corresponding norm I $I_{x}$, and the orthogonal decomposition $R_{\mu}^{n}=A_{\mu}^{n} \oplus B_{\mu}^{n}$. Let us denote by $\alpha$ and $\beta$ the orthogonal projections of $\mathbf{R}_{\mu}^{n}$ onto $\Lambda_{\mu}^{n}$ and $B_{\mu}^{n}$. We have the $n$-resolution"

(5) $0 \longrightarrow 0 \longrightarrow B_{\mu}^{0} \stackrel{B D_{0}}{\longrightarrow} B_{\mu}^{1} \stackrel{B D_{0}}{\longrightarrow} \ldots \stackrel{B D_{0}}{\longrightarrow} B_{\mu}^{n} \longrightarrow 0$

which is equivalent to the Spencer's resolution in a sense of the following

Theoren 1. The diagram 


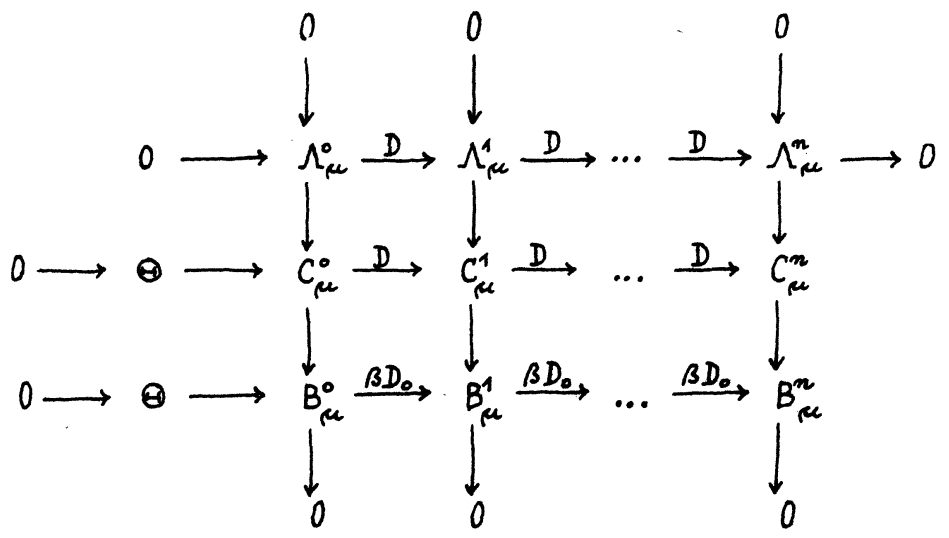

$\left(\Lambda_{\mu}^{n}=A_{\mu}^{n} \oplus A_{\mu}^{\mu+1}\right)$ is commutative, and the last row is exact if and only if the middle one is. The first row is always exact.

Definition 2. The differential operator $D: E \rightarrow E$ is said to be elliptic if for any nonzero cotangent vector $\xi \in T_{x}^{*}$ the symbol map

$$
\sigma(D, \xi): E_{x} \rightarrow F_{x}
$$

is injective at each point $x \in M$.

We can characterize the ellipticity of the operator (which is understood to be regular) $D$ in somewhat more convenient ways for our purposes.

Proposition 3. The ellipticity of an operator $D$ is equivalent to any one of this following properties:

(1) the symbol of $\omega$ in the following sequence is injective,

$$
0 \rightarrow R_{\mu} \rightarrow J_{\mu}(E) \stackrel{\omega}{\longrightarrow} J_{\mu}(E) / R_{\mu} \rightarrow 0 \text {, }
$$


i.e. for every $\xi \in T_{x}^{*}, \quad \xi \neq 0, x \in M$, the composition

$$
E_{x} \stackrel{i(\xi)}{\longrightarrow} E_{x} \otimes S^{\mu}\left(T_{x}^{*}\right) \stackrel{\gamma}{\longrightarrow} J_{\mu}(E) \stackrel{\omega}{\longrightarrow} J_{\mu}(E) / R_{\mu}
$$

is injective, where $i(\xi): e \longrightarrow e \otimes \xi^{\mu}$;

(ii) the composition

$g_{\mu} \longrightarrow E \otimes S^{\mu}\left(T^{*}\right) \longrightarrow E \otimes S^{\mu}\left(T^{*}\right) / i(\xi) E$

is injective for each $\xi$;

(iii) for each $\xi$ the composition

$$
g_{\mu} \stackrel{\sigma^{\prime}}{\longrightarrow} g_{\mu-1} \otimes T^{*} \stackrel{\xi}{\longrightarrow} g_{\mu \mu-1} \otimes \Lambda^{2} T^{*}
$$

is injective;

(iv) the sequence

$$
0 \longrightarrow \sigma\left(g_{\mu}\right) \stackrel{\xi}{\longrightarrow} \sigma^{r}\left(g_{\mu} \otimes T^{*}\right)
$$

is exact for each nonzero $\xi$.

Proof. (i) follows easily from the Definition 2.

(i) $\longleftrightarrow$ (ii) The fact that $\gamma \circ i(\xi) E_{x} \cap R_{\mu}=\varnothing$.

implies that $i(\xi) E_{x} \cap g_{\mu e}=\varnothing$. If the composition map in (ii) is not injective for some $\xi \neq 0$, and some $x \epsilon$ $\epsilon \mathbb{M}$, then $\gamma \circ i(\xi) e \in R_{\mu} I_{x}$ for some $e \in \mathbb{E}_{x}$ and then $\left.i(\xi) e \in g_{\mu}\right|_{x}$ which gives the contradiction.

$(1 i) \longleftrightarrow$ (iii) is essentially proved by the fact that the kernel of the composition map $\xi \wedge \sigma^{r}$,

$$
\begin{aligned}
& E_{x} \otimes S^{\mu}\left(T_{x}^{*}\right) \stackrel{\sigma^{\mu}}{\longrightarrow} E_{x} \otimes S^{\mu-1}\left(T_{x}^{*}\right) \otimes T_{x}^{*} \stackrel{\xi}{\longrightarrow} \\
& \stackrel{\xi}{\longrightarrow} E_{x} \otimes S^{\mu-1}\left(T_{x}^{*}\right) \otimes \Lambda^{2} T_{x}^{*}
\end{aligned}
$$

is $i(\xi) E_{x}$.

And the last statement (iv) follows from (111). 
Ellipticity of an involutive operator is characterized by

Broposition 4. The prolongati on $D_{\mu}, \mu \geq \mu_{1}$ of the operator $D$ of order $\mu_{0}$ is an involutive operator, and It is an elliptic operator if and only if the map

$$
\text { s } \sigma: \tilde{\omega}^{*} q_{\mu+1} \longrightarrow \tilde{\omega}^{*} A_{\mu}^{2}
$$

8 a oomposition of

$$
\text { s : } \tilde{\omega}^{*} A_{\mu}^{1} \longrightarrow \tilde{\omega}^{*} A_{\mu}^{2} \quad \text { and } \delta^{2}: \tilde{\omega}^{*} g_{\mu+1} \longrightarrow \tilde{\omega}^{*} A_{\mu}^{1}
$$

(where $\widetilde{\omega}^{*}: S^{*}(M) \longrightarrow M$ 1s the unit cotan-

gent sphere bundle) is injective.

This statement is a consequence of Proposition 3.

Let us denote by $a^{*}$ the (formal) adjoint to $a$ with respect to the scalar product

$$
(,)=\int\langle,\rangle d M \text {. }
$$

We can now state

Lemma 1. ( $[8]$ ) The ellipticity of an operator $D$ is equivalent to the existence of a positive number $c$ such that for each section $\xi \in A_{\mu}^{n}, \mu \geq \mu_{1}, 1 \leq \mu \leq n$, over any coordinate neighborhood $U C M$ with compact support, the following inequality holds:

(6) $\quad\left(\left\{d\left(\alpha d^{*}\right)+\left(\alpha d^{*}\right) d\right\} \xi, \xi\right) \geqslant c\|\xi\|_{(1)}-\|\xi\|^{2}$.

Proof. From Proposition 3 follows that the ellipticity of an operator $D$ is equivalent to the exactness of the short sequence

$$
O \longrightarrow A_{\mu}^{1} \stackrel{\xi}{\longrightarrow} A_{\mu}^{2}
$$


But D.G.Quillen ([6]) proved that the exactness of $0 \rightarrow$ $\rightarrow C_{\mu}^{0} \stackrel{\sigma(D, \xi)}{\longrightarrow} C_{\mu}^{1} \quad$ is equivalent to the exactness of the last row of the diagram

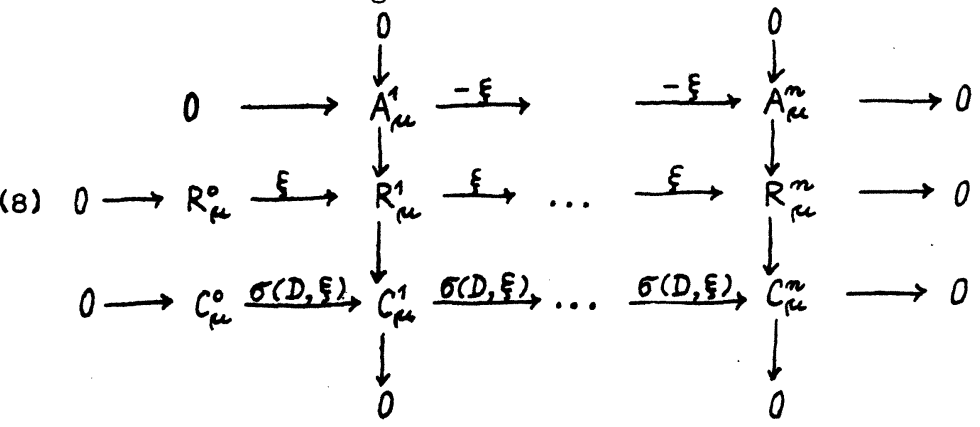

in stable range. This proves that the exactness of $(7)$ is equivalent to the exactness of the first row in (8) for all $\mu \geq \mu_{1}$. Then there exists a positive constant $c^{\prime}$ such that for each nonzero $\xi \in T_{x}^{*}$ and any $\left.\lambda \in A_{\mu}^{r}\right|_{x}, 0 \leqslant n \leqslant$ $\leqq n$, the inequality

$$
|\xi \lambda|_{x}^{2}+\left|\left(\alpha \xi^{*}\right) \lambda\right|_{x}^{2} \gtrsim c^{\prime}|\xi|_{x}^{2}|\lambda|_{x}^{2}
$$

holds at each point $x \in M$. Using Fourier transform we obtain the equivalence of $(9)$ and $(6)$.

On the basis of the " $\beta$-sequence" we get

Lemma 2. The ellipticity of an operator $D$ is equivalent to the existence of a constant $c, 0<c<1$, such that

$$
\left\|\left(\beta d^{*}\right) \xi\right\|^{2} \leq(1-c) \sum_{j}\left\|d_{j} \xi\right\|^{2}
$$

for any $\xi \in A_{\mu}^{n}, \mu \geqq \mu_{1}, \mu \geqq 1$ with compact support in $U, \sum d x^{j} \wedge d_{j} \xi=d \xi$.

Proge. Let us notice first of all that for $\mu \geqq \mu_{1}$ the exactness of the sequence 
$0 \longrightarrow B_{\mu}^{0} \stackrel{\beta \xi}{\longrightarrow} B_{\mu}^{1} \stackrel{B \xi}{\longrightarrow} B_{\mu}^{2} \stackrel{\beta \xi}{\longrightarrow} \ldots \stackrel{\beta \xi}{\longrightarrow} B_{\mu}^{n} \longrightarrow 0$

is equivalent to the ellipticity of the operator $D$. But from the diagram

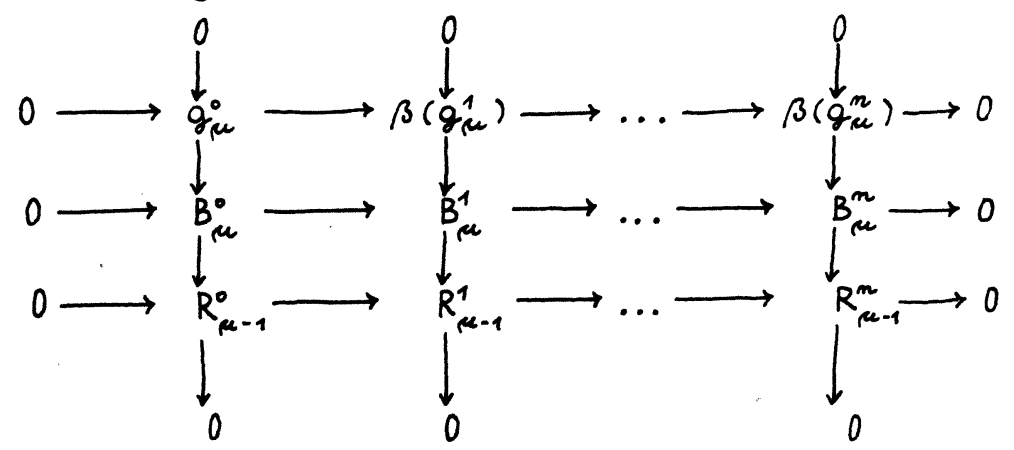

follows that the first row is exact if and only if $D$ is elliptic. And now similar argument as in the proof of Lemma. I gives the statement.

4. Before we are able to give the proof of the main result we have to consider that differential geometric aspect of the regular differential operator $D$ of order $\mu$ on the compact $n$-dimensional $C^{\infty}$-manifold $M$.

Let $\left\{\omega^{1}, \ldots, \omega^{n}\right\}$ be the orthogonal coframe in the coordinate neighborhood $U \subset M$ with respect to the chosen riemannian metric on $M$. This means that $\omega^{i} \cdot \omega^{k}=\delta_{h}^{i}$; $i$, te $=1,2, \ldots, n$. Let $\omega^{i}=a_{k}^{i} d x^{k}, d x^{i}=$ $=b_{h}^{i} \omega^{k}$, where for the $c^{\infty}$-functions holds $a_{j}^{h} b_{h}^{i}=$ $=\delta_{j}^{i}$. We shall use the symbol $\frac{\partial}{\partial \omega^{i}}$ for the cotangent vector dual to $\omega^{i}$ and also for the derivation in the exterior algebra of differential forms on $M$. Let us denote 
by $\nabla, \nabla=\omega^{i} \wedge \nabla_{i}$, the covariant differential with respect to the metric connection on $M$. We have then from the fact $\nabla \omega^{i}=0, \quad i=1,2, \ldots, m$ the identity $d \omega^{i}=\pi_{j k}^{i} \omega^{h} \wedge \omega^{j}$. The curvature form of the riemannian wetric on $M$ is the 2-form $R=\nabla(\pi)$; $\pi=\left(\pi_{j k}^{i} \omega^{k}\right)$ being the connection form. We shall use the usual star operation "* " on forms with values in a vector bundle, the symbol $\pi$ for interior product. The volume element of $M$ will be denoted by $*(1)=\omega^{1} \wedge \ldots \wedge \omega^{n}$. Let $\left\{e_{1}(x), \ldots, e_{k}(x)\right\}$ be a frame in the fibre of $A_{\mu}^{0}$ over $x \in U$, and $\left\{e_{k+1}(x), \ldots, e_{m}(x)\right\}$ a frare of $B_{\mu}^{0}$ at $x$. Then the choice of the frame $\left\{e_{1}(x), \ldots\right.$ $\left.\ldots, e_{m}(x)\right\}$ in $\left.R_{\mu}^{n}\right|_{x}$ gives a trivialization of $R_{\mu}^{\mu}$ over $U$ for all $r$, and any section $\sigma \in R_{\mu}^{r} \mid U$ can be written in the form

$$
\sigma=\frac{1}{n !} \sum_{i_{1}, \ldots, i_{n}} \sum_{\alpha} \sigma_{i_{1} \ldots i_{n}}^{\alpha} \omega^{i_{1}} \wedge \ldots \wedge \omega^{i_{n}} \otimes e_{\alpha} .
$$

The matrix $a=a(x)=\left(a_{\alpha \beta}(x)\right)$ is the matrix of the metric tensor, with components $a_{\alpha \beta}(x)=\left\langle e_{\alpha}(x)\right.$, $e_{\beta}(x)>$. Remember that the global product on $M$ is given by the formula $(\sigma, \rho)=\int_{M} \sigma \wedge a * \rho$ for any sections $\sigma, \rho$ of $R_{M}^{k}$. The 1-form $\theta=\frac{1}{2} a^{-1} d a$ is the connection form of the metric connection uniquely associated to the riemannian metric along the fibres of $R_{\mu}$. The curvature form of this connection is given by the formula $\pi=d \theta+\theta \wedge \theta$.

We have seen already that $\Lambda$ is the 1-form of connection on $R_{\mu}$, which is given by the covariant differential $D_{0}=$ $=d+e(\Lambda)$. Let us define further the operators 


$$
\begin{aligned}
D_{\Gamma}: \frac{R_{\mu}^{n}}{D_{\Gamma}}=\frac{R_{\mu}^{n+1}}{D_{0}+e(\Gamma), \quad \text { by the formula }} \\
\end{aligned}
$$

The operator $D_{r}$ is again the covariant differential of some connection on $R_{\mu}$. Considering the commutator of $D_{0}$ and $D_{\Gamma}$, we define a local 2-form $\Omega=D_{0} D_{\Gamma}+D_{\Gamma} D_{0}$. Using the orthogonal coframe introduced above, we have

$$
D_{\Gamma}=\omega^{i} \wedge D_{j}, D_{0}=\omega^{j} \wedge D_{j} \text {. }
$$

Then for each $\sigma \in R^{n}$

$$
\left(D_{j} D_{k}-D_{k} D_{j}\right) \sigma_{i_{1} \ldots i_{k}}^{\alpha}=\Omega_{n_{j h}}^{\alpha} \sigma_{i_{1} \ldots i_{n}}^{\beta} .
$$

Definition 3. The 2-form $\Omega$ is called the curvature form of the operator $D$ and the corresponding tensor the curvature tensor of $D$.

Let $D_{0}^{*}$ be the adjoint operator to $D_{0}$ with respect to the global product $($,$) . Then for \sigma \in R_{\mu}^{r}$ the Weitzenböck formula has the form

$$
\left\{\left(D_{0} D_{0}^{*}+D_{0}^{*} D_{0}\right) \sigma\right\}_{i_{1} \cdots i_{n}}^{\alpha}=-\sum_{j=1}^{n} D_{j} D_{j} \sigma_{i_{1} \ldots i_{n}}^{\alpha}+
$$

$$
\begin{aligned}
& +\sum_{\beta=1}^{N}\left(\Omega_{\beta j i_{1}}^{\alpha}-\sigma_{\beta}^{\alpha \alpha} R_{i_{1}}^{j}\right) \sigma_{i_{1} \ldots i_{\beta-1} j i_{B+1} \ldots i_{k}}- \\
& \left.-\frac{1}{2} \sum_{i_{\mu, i_{\nu 1 j}}} R_{i_{\mu} i_{\nu j}}^{e} \sigma_{i_{1} \ldots i_{\mu-1}}^{\alpha} e i_{\mu+1} \ldots i_{\nu-1} j i_{\nu+1} \ldots i_{n j}\right),
\end{aligned}
$$

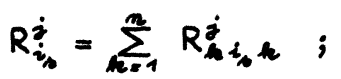

or symbolically

$$
\square_{0} \sigma=-\sum D_{j} D_{j} \sigma-\frac{1}{2} R \sigma-\hat{R} \sigma+\Omega \sigma \text {. }
$$

We shall use the notation $\Omega(\sigma, \sigma)=(\Omega \sigma, \sigma)$, $\hat{R}(\sigma, \sigma)=(\hat{R} \sigma, \sigma), R(\sigma, \sigma)=(R \sigma, \sigma)$. 
Lemma 3. For each element $\sigma \in R_{\mu}^{N}$ the foll lowing Adentits holds :

$$
\text { (12) } \sum_{j, m=1}^{m}\left(\left\{D_{j} D_{h}-D_{h} D_{j}\right\}\left(\sigma \pi \frac{\partial}{\partial \omega^{j}}\right), \sigma \pi \frac{\partial}{\partial \omega^{k}}\right)=\Omega(\sigma, \sigma)-\hat{R}(\sigma, \sigma)-\frac{1}{2} R(\sigma, \sigma) \text {. }
$$

Proof. For any $\sigma \in R_{\mu}^{n}$ we get in $U$ the formulas $\left\{D_{j} D_{k}\left(\sigma \pi \frac{\partial}{\partial \omega^{\alpha}}\right)\right\}^{\alpha}=\frac{1}{(n-1) !} \sum\left\{\sigma_{\left.j i_{1} \ldots i_{k-1}\right\} k_{j} j}^{\alpha}+\sigma_{\left.j i_{1} \ldots i_{k-1}\right) k}^{\beta} \Gamma_{\beta j}^{\alpha}\right\} \cdot$

$\cdot \omega^{i_{1}} \wedge \ldots \wedge \omega^{i_{n-1}}$,

$$
\begin{aligned}
& \left\{D_{h} D_{j}\left(\sigma \pi \frac{\partial}{\partial \omega^{j}}\right)\right\}^{\alpha}=\frac{1}{(k-1) !} \sum\left\{\sigma_{j i_{1} \ldots i_{k-1} ; j ; k}^{\alpha}+\Gamma_{\beta j ; k}^{\alpha} \sigma_{j i_{1} \ldots i_{k-1}}^{\beta}+\right. \\
& \left.+\Gamma_{\beta j}^{\alpha} \sigma_{j i_{1} \ldots i_{n-1} ; k}^{\beta}-\Lambda_{\gamma k k}^{\beta} \Gamma_{\beta \neq}^{\alpha} \sigma_{j i_{1} \ldots i_{n-1}}^{\gamma}\right\} \omega^{i_{1}} \wedge \ldots \wedge \omega^{i_{k-1}} .
\end{aligned}
$$

This proves the statement.

If $M$ is a finite submanifold of the $c^{\infty}$-manifold $M^{*}$, the closure $\bar{M}$ is compact, and the boundary bM of $M$ is regularly imbedded $C^{\infty}$-submanifold of $M^{\prime}$ of codimension 1 . Let $r(x)$ be the distance function, then we have the formulas $d r \wedge * d r=d r \cdot d r *(1)=*(1), d r=\frac{\partial r}{\partial \omega^{n}} \omega^{n}$.

We have for each $\sigma \in \underline{R}_{\mu}^{\kappa} \mid U$ the decomposition $\sigma=t \sigma+$ $+n \sigma$, where $t \sigma$ is tangential and $n \sigma$ normal at each $x \in b M$. On the basis of the formula

$$
t\left(\omega^{1} \wedge \ldots \wedge \omega^{l} \wedge \ldots \wedge \omega^{n}\right)=(-1)^{l-1} \frac{\partial r}{\partial \omega^{l}} *(d r)
$$

we get the "integration by parts".

Proposition 5. For any $C^{\infty}$-functions $a, P, B$ holds on a P1nite manifold $M$ the identity

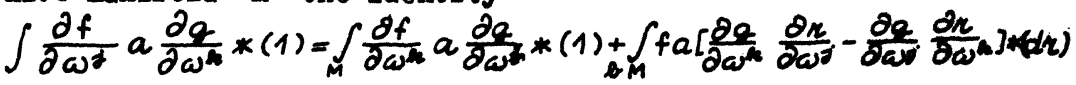

$$
\begin{aligned}
& +\int_{M} f a\left(c_{j h}^{h}-c_{h j}^{h}\right) \frac{\partial g}{\partial \omega^{h}} *(1)+\int_{M} f a\left(\frac{\partial q}{\partial \omega^{j}} S_{M}-\frac{\partial q}{\partial \omega^{h}} S_{j}\right) *(1)+ \\
& +\int_{M}\left(\frac{\partial f}{\partial \omega^{k}} \frac{\partial a}{\partial \omega^{d}} q-f \frac{\partial a}{\partial \omega^{d}} \frac{\partial q}{\partial \omega^{n}}\right) *(1) \text {. }
\end{aligned}
$$

$-189-$ 
5. The exactness of the sequence (4) or (5) is a consequence of solvability of so-called Neumann problem, which will be discussed in tinis part.

Let $D$ be an elliptic operator and $M$ a finite subimanifold as above. $D^{*}$ be the formal adjoint to the operator $D$. We use the following notation:

C : the restriction of the space of sections of $C_{\mu}=$ $=\oplus_{n} C_{\mu}^{n}$ to $\bar{M}$, eleivents which are smooth up to and including the boundary;

C. : the completion of $\mathbb{C}$ in the norm $\|$;

$D, D^{*}$ : the extension to $\mathbb{C}_{0}$ of the operators $D, D^{*}$ in Spencer's sequence;

$N$ : the elements $u \in \mathbb{C}_{0}$ such that $D \mu$ lies in the domain of $D^{*}$, and $D^{*} \mu$ lies in the domain of $D$;

H : the subspace of $N$ composed of the elements of $\mathbb{N}$ which are annihilated by the Fridrichs extension $I$ of $\square=D D^{*}+D^{*} D$ on $\mathbb{N}$.

Definition 4. We say that the $D$-Neumann problem is solvable for a finite manifold $M$, and the elliptic differential operator $D$, if $I N$ is closed in $\mathbb{C}_{0}$ and the Neumann operator (13)

$$
N: \mathbb{C}_{0} \longrightarrow N
$$

commutes with $D$.

Remark. If IN is closed in $\mathbb{C}_{0}$ we have the orthogonal decomposition $\mathbb{C}_{0}=D D^{*} \mathbb{N} \oplus D^{*} D \mathbb{N} \oplus H, \quad$ and we define the mapping (13). by the relation $N u=w-H w$, where $H: \mathbb{C}_{0} \longrightarrow \mathbb{H}$ is an orthogonal projection and 


$$
\mu=L w+H w, \quad w \in N \text {. }
$$

Proposition 6. The D-Neumann problem is solvable if and only if the operators $H$ and $N$ have the following properties: $N$ is a self-adjoint bounded operator satisfying $\mathrm{NH}=\mathrm{FN}=0$,

$$
\mu=D D * N \mu+D^{*} D N \mu+H \mu
$$

for each $u \in \mathbb{C}_{0}$, and

$$
N D=D N
$$

Proof. Follows essentially the lines of the proof of Proposition 2.8 ( $[5])$.

From the work of J.J.Kohn and L.Nirenberg follows that if

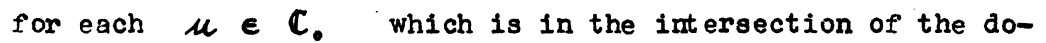
mains of $D$ and $D^{*}$ holds for some $\varepsilon>0$ the inequality

(14) $\quad(D \mu, D \mu)+\left(D^{*} \mu, D^{*} \mu\right)+(\mu, \mu) \geq \varepsilon \int_{b}|\mu|_{x}^{2} *(d x)$ then the D-Neumann problem is solvable and

$$
H \cong Z\left(\mathbb{C}_{0}\right) / D\left(\mathbb{C}_{0}\right) \text {. }
$$

The solvability of the D-Neumann problem implies the isomorphis $\mathrm{m} \boldsymbol{H}^{n} \cong \mathbb{H}^{n}$, where $\boldsymbol{H}=\oplus_{n} \boldsymbol{H}^{n}$ is the space of elements $\mu \in \mathbb{C}$, which satisfy the conditions

$$
\begin{aligned}
& \left(D^{*} u, v\right)=\left(u, D_{v}\right), \\
& \left(D^{*} D_{u, v}\right)=\left(D_{u}, D_{v}\right)
\end{aligned}
$$

for all $v \in \mathbb{C}$, and which are annihilated by the laplacian $\square$.

Spencer's confecture. (The local Neumann problem): If $D_{\mu}$ is an elliptic operator, then the D-Neumann problem is solvable on sufficiently small spherical neighborhood of euclidean n-space and, for these domains $H^{n}=0$ for $n \geq 1$. 
The problem then remains, to prove an estimate of the form (14).

6. The harmonic space $\boldsymbol{H}$ is the subspace of $\mathbb{C}$, which elements satisfy both boundary conditions and whic $h$ are annihilated by the laplacian $\square$. For the fundamental eotimate is essential the explicit expression of the Dirichlet integral, when the elements $u \in \mathbb{C}$ are smooth up to and Including the boundary and satisfy only the first boundary condition $\left(D^{*} u, v\right)=\left(u, D_{v}\right)$ for all $v \in \mathbb{C}$.

Let us use the notation

$$
\begin{aligned}
& \alpha_{k j}=\left(\Lambda_{k}+\tilde{\Lambda}_{k}+\tilde{\Gamma}_{k}-\tilde{\theta}_{k}\right) D_{j}-\left(\tilde{\Lambda}_{j}+\Lambda_{j}+\Gamma_{j}-\tilde{\theta}_{j}\right) D_{k}, \\
& \tilde{\Lambda}=a \Lambda a^{-1}, \tilde{\Gamma}=a \Gamma a^{-1}, \tilde{\theta}=a \theta a^{-1}, \alpha_{k}=\alpha D_{k}-D_{k} \alpha, \\
& \tilde{\alpha}_{h}=\alpha D_{k}-D_{k} \alpha, L_{i j}=\nabla_{j} \nabla_{i} n .
\end{aligned}
$$

We say that the finite manifold $M$ is "strongly pseudoconvex" if at each point of bM holds

$$
\sum_{i, j=1}^{n} L_{i j}\left\langle\alpha\left(\xi \pi \frac{\partial}{\partial \omega^{i}}\right), \alpha\left(\xi \pi \frac{\partial}{\partial \omega^{j}}\right)\right\rangle>0 .
$$

The quadratic forms $\widetilde{\Omega}(\xi, \xi), \hat{\Re}(\xi, \xi), \mathcal{R}(\xi, \xi)$ are deeined by the formula:

$$
\begin{aligned}
\widetilde{\Omega}(\xi, \xi) & -\hat{R}(\xi, \xi)-\frac{1}{2} R(\xi, \xi)= \\
& =\sum_{j, h}\left(\left\{D_{j} D_{h}-D_{h} D_{j}\right\} \propto\left(\xi \pi \frac{\partial}{\partial \omega^{j}}\right), \propto\left(\xi \pi \frac{\partial}{\partial \omega^{h}}\right)\right) .
\end{aligned}
$$

Lemmen 4. For any $\mu \in \mathbb{C}$ which satisfies the first boundary condition (15), we have the following identity:

$$
\|D \mu\|^{2}+\left\|D^{*} u\right\|^{2}+\|\mu\|^{2}=K(u, \mu)+T(u, u)+\int_{b} L(\mu, \mu) *(d r) \text {, }
$$

where 
$K(\mu, \mu)=\Omega(\sigma, \sigma)-\hat{R}(\sigma, \sigma)-\frac{1}{2} R(\sigma, \sigma)+\widetilde{\Omega}(\xi, \xi)-\hat{\mathcal{R}}(\xi, \xi)-$ $-\frac{1}{2} R(\xi, \xi)$,

$L(\mu, \mu)=\sum_{i, j=1}^{n} L_{i j}\left\langle\alpha\left(\xi \pi \frac{\partial}{\partial \omega^{i}}\right), \alpha\left(\xi \pi \frac{\partial}{\partial \omega^{y}}\right)\right\rangle+\sum_{i, j=1}^{n} L_{i j}\left\langle\sigma \pi \frac{\partial}{\partial \omega^{2}} ;\right.$ $\left.\sigma \pi \frac{\partial}{\partial \omega^{\delta}}\right\rangle$

$T(\mu, \mu)=\sum_{k=1}^{n}\left\|D_{h} \sigma\right\|^{2}+\sum_{k=1}^{n}\left\|D_{k} \xi\right\|^{2}-\left(\beta D_{j}\left(\xi \pi \frac{\partial}{\partial \sigma k}\right), \beta D_{k}\left(\xi \pi \frac{\partial}{\partial \sigma \partial}\right)\right)+$ $+\left(d_{n f}\left(\sigma \pi \frac{\partial}{\partial \omega^{j}}\right), \sigma \pi \frac{\partial}{\partial \omega^{\mu}}\right)+\left(S_{n} D_{i}\left(\sigma \pi \frac{\partial}{\partial \omega^{\pi}}\right), \sigma \pi \frac{\partial}{\partial \omega^{2}}\right)-$ $-2\left(D_{j} \sigma \pi \frac{\partial}{\partial \omega^{\mu}}, \sum C_{l_{j}}^{e}\left(\sigma \pi \frac{\partial}{\partial \omega^{e}}\right)\right)-\left(D_{j}\left(\sigma \pi \frac{\partial}{\partial \omega^{z}}\right), S_{k}\left(\sigma \pi \frac{\partial}{\partial \omega^{\pi}}\right)\right)+$ $+\left(d_{\text {nj }} \propto\left(\xi \pi \frac{\partial}{\partial \omega^{z}}\right), \alpha\left(\xi \pi \frac{\partial}{\partial \omega^{n}}\right)\right)+\left(S_{n} D_{z} \propto\left(\xi \pi \frac{\partial}{\partial \omega^{n}}\right), \alpha\left(\xi \pi \frac{\partial}{\partial \omega^{j}}\right)\right)-$ $-2\left(D_{j} \xi \pi \frac{\partial}{\partial \omega^{h}}, \sum c_{k j}^{e}\left(\xi \pi \frac{\partial}{\partial \omega^{2}}\right)\right)-\left(D_{j} \propto\left(\xi \pi \frac{\partial}{\partial \omega^{j}}\right), S_{n} \propto\left(\xi \pi \frac{\partial}{\partial \omega^{2}}\right)\right)-$ $-2\left(D_{j} \alpha\left(\xi \pi \frac{\partial}{\partial \omega^{k}}\right), \alpha_{h}\left(\xi \pi \frac{\partial}{\partial \omega^{j}}\right)\right)+\left(D_{n} \alpha\left(\xi \pi \frac{\partial}{\partial \omega^{k}}\right), \alpha_{j}\left(\xi \pi \frac{\partial}{\partial \omega^{g}}\right)\right)-$ $-\left(\sum c_{j h}^{h}\left(\sigma \pi \frac{\partial}{\partial \omega^{h}}\right), \sum c_{h j}^{l}\left(\sigma \pi \frac{\partial}{\partial \omega^{e}}\right)\right)-\left(\sum c_{j h}^{h}\left(\xi \pi \frac{\partial}{\partial \omega^{h}}\right)\right.$, $\left.\sum c_{k j}^{e}\left(\xi \pi \frac{\partial}{\partial \omega^{\ell}}\right)\right)-$ $-\left(\alpha_{j}\left(\xi \pi \frac{\partial}{\partial \omega^{h}}\right), \alpha_{m}\left(\xi \pi \frac{\partial}{\partial \omega^{2}}\right)\right)+\left(\tilde{\alpha}_{j}\left(\xi \pi \frac{\partial}{\partial \omega^{j}}\right), \tilde{\alpha}_{m}\left(\xi \pi \frac{\partial}{\partial \omega^{n}}\right)\right)+$ $+\|\sigma\|^{2}+2\|\xi\|^{2}+\left\|D_{0}^{2} \sigma\right\|^{2}+\left\|D_{0}^{* 2} \xi\right\|^{2}+\|\alpha \sigma\|^{2}+2\left\{\left(D_{0}^{*} \sigma, D_{0}^{* 2} \xi\right)+\left(\alpha \sigma, \alpha D_{0}^{*} \xi\right)\right.$ $\left.\left.-\left(\xi, D_{0} \sigma\right)-\left(D_{0}\right\}, D_{0}^{2} \sigma\right)\right\}-\int_{j} \frac{\partial}{\partial \omega^{\omega}}\left\langle\pi_{j}\left(\sigma \pi \frac{\partial}{\partial \omega^{2}}\right), \sigma \pi \frac{\partial}{\partial \omega^{\gamma}}\right\rangle *(1)-$ $-\int_{M} \frac{\partial}{\partial \omega^{j}}\left\langle\pi_{j} \propto\left(\xi \pi \frac{\partial}{\partial \omega^{n}}\right), \propto\left(\xi \pi \frac{\partial}{\partial \omega^{\delta}}\right)\right\rangle *$ (1).

Theorem 2. If the curvature of the elliptic operator $D$ 1s such that the quadratic form $\mathrm{K}(u, u)$ is sufficiently positive for all $u \in \mathbb{C}^{n}, n \leq 1$, and 1 both conditions 
(15) and condition (16) are satiailed, then $H^{n}=0, r \geqslant 1$. Eroof. Investigation of the expression

$$
K(\mu, \mu)+T(\mu, \mu)+\int_{n} L(\mu, \mu) *(d r)=\|\mu\|^{2}
$$

gives the statecient.

The cain result concerns the cohoriology Eroups $H^{n}$ for a compact manifold, assuaing that $D$ is an elliptic operator. On the basis of the resolution (4) and (5) (which is assumod to be exact) of the sheaf $\Theta$, and using de Tham's and Hodge's theorem we conclude that it is enough to investigate the harmonic ele ients of the graded vector space $C=\oplus_{n} C^{n} \quad$ of sections of $C_{\mu}=\oplus_{n} C_{\mu}^{\mu} \quad$ over the manifold $U$. We get

Lemma 5 . If $D$ is an elliptic operator, then for any harmonic jet form $\mu=(\sigma, \xi) \in C^{n}, \quad \geqq 1$, there exist positive constants $K_{1}, K_{2}$ such that

$$
K(u, u) \leq K_{1}\|\sigma\|^{2}+K_{2}\|\xi\|^{2},
$$

where

$$
\begin{aligned}
K(\mu, \mu) & =\Omega(\sigma, \sigma)-\frac{1}{2} R(\sigma, \sigma)+\widetilde{\Omega}(\xi, \xi)-\frac{1}{2} R(\xi, \xi)- \\
& -\hat{R}(\sigma, \sigma)-\hat{R}(\xi, \xi) .
\end{aligned}
$$

Proof. Let us notice first of all that for any harmonic jet form $\mu(\sigma, \xi) \in C^{n}, r \geqslant 1$, where $(\sigma, \xi)$ is an obvious representation of $u$, we get the formulas

$$
\begin{aligned}
& \Omega(\sigma, \sigma)-\frac{1}{2} R(\sigma, \sigma)-\hat{R}(\sigma, \sigma)=-\sum_{j}\left\|D_{j} \sigma\right\|^{2}+\cdots, \\
& \tilde{\Omega}(\xi, \xi)-\frac{1}{2} R(\xi, \xi)-\hat{R}(\xi, \xi)=-\sum_{j}\left\|D_{j} \xi\right\|^{2}+\left\|_{\beta} D_{0}^{*} \xi\right\|^{2}+\cdots,
\end{aligned}
$$

where - . donotes the half order terms. From Lemma 2 we have the formula. 


$$
\begin{aligned}
& \left\|\beta D_{0}^{*} \xi\right\|^{2}-\sum_{j}\left\|D_{j} \xi\right\|^{2}=-\sum_{j}\left\|d_{j} \xi\right\|^{2}+\left(\beta d_{h}\left(\xi \pi \frac{\partial}{\partial x^{h}}\right),\right. \\
& \left.\beta \alpha_{h}\left(\xi \pi \frac{\partial}{\partial x^{j}}\right)\right)+\cdots .
\end{aligned}
$$

Applying these identities to the forrula for $k(u, u)$, we get the statement after some investigation.

Theorem 3. Let $M$ be a compact manifold and $D$ an elliptic differential operator. If the quadratic form $\mathrm{K}(\mathrm{u}, \mathrm{u})$ is sufficiently positive for al $\mu \in C^{n}, n \geq 1$, then $H^{n}(M, \Theta)=0, \quad \kappa \geq 1$.

Considering the $\beta$-resolution of the sheaf $\Theta$ we get another form. The cohomology $H(M, \Theta)$ is isomorphic to the space $B=\oplus_{n} B^{n}$ of sections of $B_{\mu}=\oplus_{n} B_{\mu}^{n} \quad$ over $M$, which are annihilated by the laplacian $D_{0}^{*} \beta D_{0}+\beta D_{0} D_{0}^{*}$. We have analogy of the Weitzenböck formula. Let

$$
K_{\beta}(\rho, \rho)=\left(\left\{D_{j} D_{n}-D_{n} D_{j}\right\} \beta\left(\rho \pi \frac{\partial}{\partial \omega^{j}}\right), \beta\left(\rho \pi \frac{\partial}{\partial \omega^{h}}\right)\right),
$$

and

$$
K_{\beta}(\rho, \rho)=\Omega_{\beta}(\rho, \rho)-\frac{1}{2} R_{\beta}(\rho, \rho)-\hat{R}_{\beta}(\rho, \rho) \text {. }
$$

Then by similar reasoning to that one we have made earlier the following statement can be proved.

Theorem 4. Let $M$ be a compact manifold and $D$ an elliptic operator. If the quadratic form $\Omega_{\beta}(\rho, \rho)$ is sufficientiy positive in the sense that $K_{\beta}(\rho, \rho)$ is sufficiently positive, for any $\rho \in B^{n}, n \geqslant 1$, then for $n \geqslant 1$ $H^{n}(M, \Theta)=0$.

It can be proved that the Kodaira's vanishing theorem is a special case of these statements. 
Let us asoume that $\mathbf{E}$ and $\mathbf{F}$ are differentiable complex vector bundles over a compact differentiable manifold $M$, and the elliptio differential operator $\mathfrak{D}: \underline{E} \rightarrow \underline{F}$ as above. We can state then

Theorem 5. If the quadratic form $K(u, u)$ is sufficiently positive for all $\mu \in C^{*}, r \neq 1$, then $\operatorname{dim} \mathbb{H}^{\circ}(M, \theta)$ is equal to the topological index of the differential operator $D+D^{*}$.

Proof. Let us consider

$$
C_{0}=\oplus_{n} C^{2 n}, \quad C_{1}=\oplus_{n} C^{2 n+1} .
$$

Then

$$
D+D^{*}: C_{0} \longrightarrow C_{1},
$$

and the adjoint operator $D+D^{*}$ maps $C_{1}$ into. $C_{0}$. The Euler-Poincare characteristic $\chi(M, \Theta)=\sum_{n=0}^{n}(-1)^{n} \cdot \operatorname{dim} H^{n}(M, \Theta)$ is the analytic index of $D+D^{*}$. And by the Atiyah-Singer theorem this is equal to the topological index of the operator $D+D^{*}$. This gives the statement.

$$
\text { References }
$$

[1] R. BOTT: Notes on the Spencer resolution, mimeographed notes, Harvard Uni versity, 1963.

[2] P.A. GHC PFITHS: Hermittan differential geometry and the theory of positive and ample holomorphic vector bundles, Journal of Math. and Mech.,14(1965),117140.

[3] L. HORANDER: Linear partial differential operators, Springer-Verlag,Berlin,ig63. 
[4] K. KODAIRA: On a differential-geometric method in the theory of analytic atacks,Proc.Nat.Acad.Sci., 39(1953), 2268-1273.

[5] J.J. KOHN: Harmonic integrals on strongly pseudoconvex manifolds, I, Ann. of Math. ,77(1963).

[6] D.G. QUILLEN, Formal properties of over-determined systems of linear partial differential equations, Harvard thesis, 1964 (to appear).

[7] I.M. SINGER and S. STERNBERG: The infinite groups of Lie and Cartan, Part I, (The transitive groups), Journal d'Anal.Math., XV (1965), 1-114.

[8] D.C. SPENCER, Deformation of structures on manifolda defined by transitivie, continuous pseudogroups III,Ann. of Math. ,81(1965), 389-450.

[9] W.J. SWEENEY: The D-Neumann problem,Stanford thesis,1966 (to appear).

(Received December 30, 1966) 